\title{
Concordance study of PD-L1 expression in primary and metastatic bladder carcinomas: comparison of four commonly used antibodies and RNA expression
}

\author{
Maria Tretiakova ${ }^{1}$, Regan Fulton ${ }^{2}$, Masha Kocherginsky ${ }^{3}$, Thomas Long ${ }^{1}$, Cigdem Ussakli ${ }^{1}$, \\ Tatjana Antic ${ }^{4}$ and Allen Gown ${ }^{2}$ \\ ${ }^{1}$ Department of Pathology, University of Washington Medical Center, Seattle, WA, USA; ${ }^{2}$ PhenoPath \\ Laboratories, Seattle, WA, USA; ${ }^{3}$ Department of Preventive Medicine, Northwestern University Feinberg \\ School of Medicine, Chicago, IL, USA and ${ }^{4}$ Department of Pathology, University of Chicago Medical Center, \\ Chicago, IL, USA
}

\begin{abstract}
Therapy with anti-PD-L1 immune check-point inhibitors is approved for several cancers, including advanced urothelial carcinomas. PD-L1 prevalence estimates vary widely in bladder cancer, and lack of correlation between expression and clinical outcomes and immunotherapy response may be attributed to methodological differences of the immunohistochemical reagents and procedures. We characterized PD-L1 expression in 235 urothelial carcinomas including 79 matched pairs of primary and metastatic cancers using a panel of four PD-L1 immunoassays in comparison with RNAscope assay using PD-L1-specific probe (CD274). The antibody panel included three FDA-approved clones (22C3 for pembrolizumab, 28.8 for nivolumab, SP142 for atezolizumab), and a commonly used clone E1L3N. Manual scoring of tissue microarrays was performed in each of 235 tumors (624 tissue cores) and compared to an automated image analysis. Expression of PD-L1 in tumor cells by $\geq 1$ marker was detected in 41/142 (28.9\%) primary tumors, $13 / 77$ (16.9\%) lymph nodes, and 2/16 (12.5\%) distant metastases. In positive cases, high PD-L1 expression ( $>50 \%$ cells) was detected in $34.1 \%$ primary and $46.7 \%$ metastases. Concordant PD-L1 expression status was present in 71/79 (89.9\%) cases of matched primary and metastatic urothelial carcinomas. PD-L1 sensitivity ranked from highest to lowest as follows: RNAscope, clone 28.8, 22C3, E1L3N, and SP142. Pairwise concordance correlation coefficients between the four antibodies in 624 tissue cores ranged from 0.76 to 0.9 for tumor cells and from 0.30 to 0.85 for immune cells. RNA and protein expression levels showed moderate to high agreement (0.72-0.87). Intra-tumor expression heterogeneity was low for both protein and RNA assays (interclass correlation coefficients: $0.86-0.94$ ). Manual scores were highly concordant with automated Aperio scores (0.94-0.97). A significant subset of 56/235 (23.8\%) urothelial carcinomas stained positive for PD-L1 with high concordance between all four antibodies and RNA ISH assay. Despite some heterogeneity in staining, the overall results are highly concordant suggesting diagnostic equivalence of tested assays.

Modern Pathology (2018) 31, 623-632; doi:10.1038/modpathol.2017.188; published online 22 December 2017
\end{abstract}

A highly promising and rapidly emerging approach to cancer immunotherapy is antibody-mediated blockade of inhibitory co-receptors expressed on T-lymphocytes, or so-called immune checkpoints. Programmed cell death-1 (PD-1; CD279) is one of immune check-point receptors of T-cells and its interaction with the PD-1

Correspondence: Dr MS Tretiakova, MD, PhD, Department of Pathology, University of Washington Medical Center, 325 9th Avenue, Seattle, WA 98104, USA.

E-mail: mariast@uw.edu

Received 21 July 2017; revised 31 October 2017; accepted 4 November 2017; published online 22 December 2017 receptor-ligand (PD-L1; CD274) is a major pathway hijacked by tumors to suppress immune control. ${ }^{1-3}$ The PD-L1trans-membrane protein is normally expressed at very low levels in peripheral tissues with a few exceptions like placental trophoblast, tonsil, and hematopoietic cells where increased PD-1/PD-L1 signaling dampens unwarranted T-cell function and autoimmunity. ${ }^{4-6}$ However, a variety of cancers show increased membranous expression of these T-cell inhibitors, thus binding of either PD-1 or PD-L1 unmasks tumor cells leading to their lysis by activated T-cells. 
Immunotherapy aimed at inhibiting PD-1/PD-L1 has been recently approved and successfully utilized for several cancers including bladder cancer. ${ }^{3,7-10}$ Yet, factors associated with benefit from such therapy remain under investigation. As PD-1/PD-L1 tissue expression could be established by immunohistochemistry, it is logical to assume their role as predictive biomarkers allowing patient selection for enriched therapy benefit. ${ }^{2,11}$ Recently several antiPD-L1 assays were developed and approved as companion and/or complementary immunohistochemical assays for specific drugs and tumor types using different antibodies, staining platforms, scoring guidelines, and definitions of PD-L1 positivity ranging from any expression to $50 \% .^{8,12}$ PD-L1 expression testing is highly regulated and limited with FDA approval requiring particular application, assay, testing platform, and specific scoring recommendations tailored to different antibody clone, tissue types, and associated drug. ${ }^{1,13}$ This poses a logistical problem for the diagnostic laboratories in offering PD-L1 testing, its interpretation, and predicting treatment response. ${ }^{6,14}$ Moreover, it raises a question of different assays compatibility, sensitivity and specificity given a common target and similar mechanism of action for anti-PD-1/PD-L1 drugs.

We aimed to establish prevalence, extent, and concordance of PD-L1 expression in a large cohort of primary and metastatic bladder cancer based on four most commonly used antibodies including a platformindependent clone E1L3N, and 3 FDA-approved clones: 22C3 (Keytruda), 28.8 (Opdivo), and SP142 (Tecentriq). We also evaluated methodological accuracy of protein detection by comparison with RNAscope assay using manual and automated scoring.

\section{Materials and methods}

\section{Case Selection and Tissue Microarrays}

This study was conducted with approvals from the University of Washington and The University of Chicago institutional review boards and included 235 tumors from 156 patients obtained from pathology archives from 2004 thought 2012. High-density tissue microarrays were constructed using 63 primary urothelial carcinomas, 74 urothelial carcinomas with matched primary tumor and regional lymph node metastases, and additional 16 distant metastases with 5 matching primary urothelial carcinomas. Each tumor was represented by two to six $1 \mathrm{~mm}$ cores (total of 624 cores, mean $=4$ cores per patient): $1-3$ cores from the primary tumor and 1-6 cores from metastases. The 101 patients with available demographic data were $65 \%$ male, with age ranging from 25 to 89 years $($ mean $=67.9)$. Tumor size ranged from 1 to $10 \mathrm{~cm}($ mean $=4.3 \mathrm{~cm})$ with the following histology: pure urothelial $(n=136)$ and mixed urothelial with variant morphologies $(n=20)$ including micropapillary $(n=11)$, squamous differentiation $(n=2)$, glandular $(n=1)$, squamous and glandular $(n=1)$, plasmacytoid $(n=1)$, signet ring $(n=1)$, and adenocarcinoma $(n=3)$.

\section{Immunohistochemistry and Manual Scoring}

All immunostains were performed at PhenoPath reference laboratory (CLIA certified, CAP certified). Tissue microarray slides were stained with 4 antiPD-L1 monoclonal antibodies using same protocols as for clinical cases and strictly following applicable manufacturer/FDA guidelines in retrieval, automated platforms, detection systems, controls, and interpretation (Supplementary Table 1). Three antibodies were FDA approved as In-Vitro-Diagnostic tests class III as complementary or companion diagnostic assays and one antibody (clone E1L3N, Cell Signaling) is a Research-Use-Only reagent. Positive controls included cell lines (PD-L1 positive MCF-7; PD-L1 negative NCI-H226, DAKO Pharm Dx), and positive normal tissues including placentas and tonsils (Supplementary Figure 1). All tissue microarrays were also stained with histiocytic marker CD163 to assure interpretation of membranous staining specific to tumor cells.

Tissue microarray slides were independently manually scored by two pathologists with $>10$ years of expertise in genitourinary pathology, immunohistochemistry, and molecular pathology (MT, RF). Discordant cases $(\sim 10 \%)$ with minor disagreement due to borderline expression levels or abundant inflammatory cells were reviewed and consensus was achieved on all cases. For comparison purposes all four antibodies were scored for \% membranous positivity of viable tumor cells and staining intensity (low and high). Multiplication of \% positivity and average staining intensity yielded a modified $H$-score on a scale of $0-200$ ( $0=$ negative, $1-49=$ low positive, 50-200 = high positive). In addition, expression of SP142 was analyzed in tumor-infiltrating immune cells for percent positivity (SP142-IC score) as per manufacturer recommendations and compared to manually scored tumor-infiltrating immune cells by three other markers.

\section{Automated Scoring}

Automated image analysis was performed on Aperio platform using a modified HER2 membranous staining algorithm. All tissue microarray slides were scanned on Aperio AT2 Scanner (Leica Biosystems/Aperio, Buffalo Grove, IL, USA), which automatically creates the high-resolution digital files. Images were then opened in web-based Spectrum Plus digital slide manager and segmented to multiple layers of tumor containing areas within each individual tissue core. The settings of membrane scoring algorithm (color thresholds, cell size, roundness, completeness, elongation, etc) were tuned to allow identification of all PD-L1 stained tumor cells. 
Region of interest within each tissue microarray spot contained representative tumor with at least 100 cells (Supplementary Figure 2). The most important data output parameters included total number of analyzed tumor cells (mean $=211$ per core), number of positive tumor cells, average membrane staining intensity, and calculated composite score on a scale of 0-200 for comparison with manual scores.

\section{In Situ RNA Detection and Scoring}

RNAscope manual procedure was performed at PhenoPath laboratories following standard protocol as previously described with minor modifications. ${ }^{15}$ In brief, tissue microarray slides were baked in dry oven for $1 \mathrm{~h}$, soaked in xylene twice for $5 \mathrm{~min}$ each, incubated for $1 \mathrm{~min}$ in 100\% ethanol twice and air dried. Pre-treatment of slides was done by boiling for 15 min in RNAscope target retrieval buffer solution and protease digestion in HybEx oven at $40^{\circ} \mathrm{C}$ for $30 \mathrm{~min}$ with multiple washes. PD-L1-specific probe (CD274) obtained from Advanced Cell Diagnostics (Newark, CA; cat\#600861) was applied for $2 \mathrm{~h}$ at $40^{\circ} \mathrm{C}$ in $\mathrm{HybEx}$ oven. After washes this step was followed by signal amplification steps, signal detection using FAST RED RNAscope 2.5 HD Detection reagent, hematoxylin counterstaining, slide clearing, and coverslip mounting.

Scoring of the RNAscope PD-L1 assay signals was performed manually by two pathologists (MT, RF) with consensus achieved in all cores. The numbers of signals (dots) were assessed on tumor cells only and interpreted according to manufacturer recommendations (Advanced Cell Diagnostics). The RNAscope scoring was done as follows: 0 if no staining or $<1$ dot/10 tumor cells; 1 in 1-3 dots/cell, 2 in 4-9 dots/cell, 3 in 10-15 dots/cell, and 4 in $>15$ dots/ cell (Supplementary Figure 1). A composite RNAscope score was calculated similarly to immunohistochemical $H$-score as a percentage of positive tumor cells multiplied by average staining score.

\section{Statistical Analysis}

Descriptive statistics were used to summarize expression by core. Each core was classified as positive ( $>0$ expression) or negative (0 expression). Tumors with multiple cores were classified as positive if any cores were positive. Patients with multiple tumors were classified as positive if any of the patient's cores were positive. Continuous variables were compared between groups using a twosample $t$-test, and categorical variables were compared using the $\chi^{2}$ test. Overall survival was defined as time from diagnosis to death from any cause. Overall survival was estimated using the method of Kaplan-Meier, and compared between groups using the log-rank test. Hazard ratios were estimated using the Cox proportional hazards model. Agreement between assay expression in all cores was assessed using Lin's concordance correlation coefficient ${ }^{16}$ and calculated R package epiR. ${ }^{17,18}$ Intraclass correlation coefficient was used to summarize the inter-assay reliability among multiple cores per patient or per tumor, and was estimated using intraclass correlation coefficient package in R. ${ }^{19}$

\section{Results}

We analyzed 624 cores from 235 distinct tumors (primary, lymph node or distant metastases) that came from 156 patients. PD-L1 expression was positive by $\geq 1$ marker in 56/235 (23.8\%) of all tumors: $41 / 142$ (28.9\%) primary, $13 / 77$ (16.9\%) lymph nodes, and 2/16 (12.5\%) distant metastases. PD-L1 expression presence by $\geq 2$ markers was detected in 18.7\% urothelial carcinoma cases including 21.1 primary, 15.6 LN, and $12.5 \%$ distant mets. In positive urothelial carcinoma cases, high PD-L1 expression ( $>50 \%$ cells) was detected in 37.5 cases: $34.1 \%$ in primary and $46.7 \%$ in metastases. Complete concordance for PD-L1 expression by all four antibodies was achieved in $85.3 \%$ cases: $80.6 \%$ in primary urothelial carcinomas and 92\% in metastases. Analysis of matched primary and metastatic urothelial carcinomas showed concordant PD-L1 expression status in $71 / 79(89.9 \%)$ cases. Tissue microarray cores analysis showed complete concordance by all 4 antibodies in 562/624 (90.1\%) cores with $291 / 345(84.3 \%)$ concordance in primary urothelial carcinomas, 223/231 (96.5\%) concordance in lymph nodes and 48/48 (100\%) concordance in distant metastases (Figure 1).

\section{Association of PD-L1 Expression, Clinico-Pathological Features, and Overall Survival}

Demographic information, tumor characteristics, and follow-up data were available for 101 of 156 patients. None of the patients were treated with anti-PD-1 or anti-PD-L1 check-point inhibitors. Clinicopathological features were not found to be significantly associated with PD-L1 positivity in tumor cells by $\geq 1$ marker or in immune cells with SP142-IC (Table 1). There were 16 deaths, with median followup of 11 months (range 2-127). PD-L1 staining in tumor cells by $\geq 1$ was associated with improved survival ( $\mathrm{HR}=0.26$ (95\% CI: 0.14-1.13); $P=0.0585$, log-rank test) (Figure 2), with nearly identical estimates for positive staining with $\geq 2$ markers $(\mathrm{HR}=0.39$ (95\% CI: 0.14-1.15); $P=064$, log-rank test). PD-L1 staining in immune cells (SP142-IC) was associated with overall survival ( $\mathrm{HR}=0.25(95 \%$ CI: 0.06-1.11); $P=0.0463$, log-rank test).

\section{Comparison of Four Immunohistochemical and RNA ISH Assays for Sensitivity}

Frequency of PD-L1 expression in tumor cells (\% positivity) was similar across assays, with the highest 
rates for clone $28.8(18.6 \%)$ and RNA ISH (20.5\%) (Supplementary Table 2). Among the 132 cores with $\geq 1$ positive marker, staining intensity multiplied by $\%$ positivity (H-score) as a measure of assay sensitivity was (ranked from highest to lowest) as follows: RNA ISH (64.6 \pm 60$), 28.8$ (54.8 \pm 66$), 22 \mathrm{C} 3$ $(43.5 \pm 63), \quad$ E1L3N $\quad(37.1 \pm 55.7)$ and SP142 $(32.6 \pm 57.1) . H$-scores for immunoassays were higher in distant metastases (range: 123.3-160) than in primary tumors (range: 33.1-53.1), and were lowest in lymph node metastases (range: 12.8-38.9).

\section{Concordance of Four Chromogenic Immunohistochemical Assays}

Pairwise comparison of tumor cell staining by four antibodies showed high agreement between markers (Table 2a). Concordance correlation coefficients ranged from 0.76 to 0.90 for all cores, and from 0.69 to 0.86 for cores positive by $\geq 1$ marker. The highest agreement was reached between $22 \mathrm{C} 3 / 28.8$ and 28.8/E1L3N pairs, whereas the lowest agreement was seen between 22C3/SP142 and 28.8/SP142,
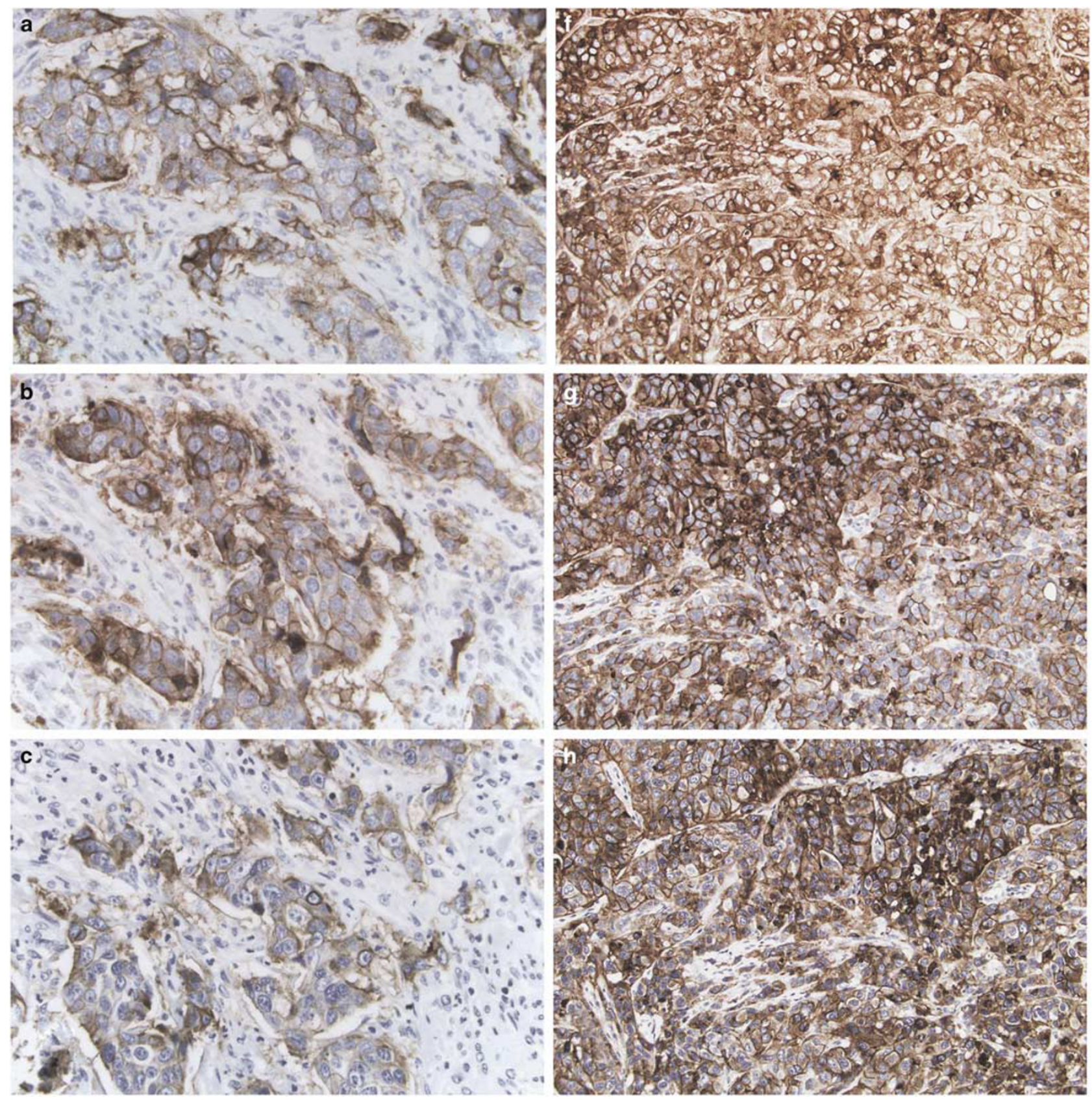

Figure 1 Representative examples of PD-L1 staining of primary and metastatic urothelial carcinomas using panel of four immunostains and RNA in situ hybridization (RNAscope) assay. (a-e) Primary urothelial carcinoma with intermediate-high PD-L1 expression levels, tissue microarray scores range: $70-150$, magnification $\times 400$. ( $\mathbf{f}-\mathbf{j})$ Metastatic to lung urothelial carcinoma strongly positive for PD-L1 expression with tissue microarray scores uniformly 200, magnification $\times 200$. Immunohistochemical staining is preformed using clones 22C3 a, f, 28.8 b, g, E1L3N c, h, and SP142 d, i; RNA ISH results using PD-L1-specific probe CD274 e, j. 

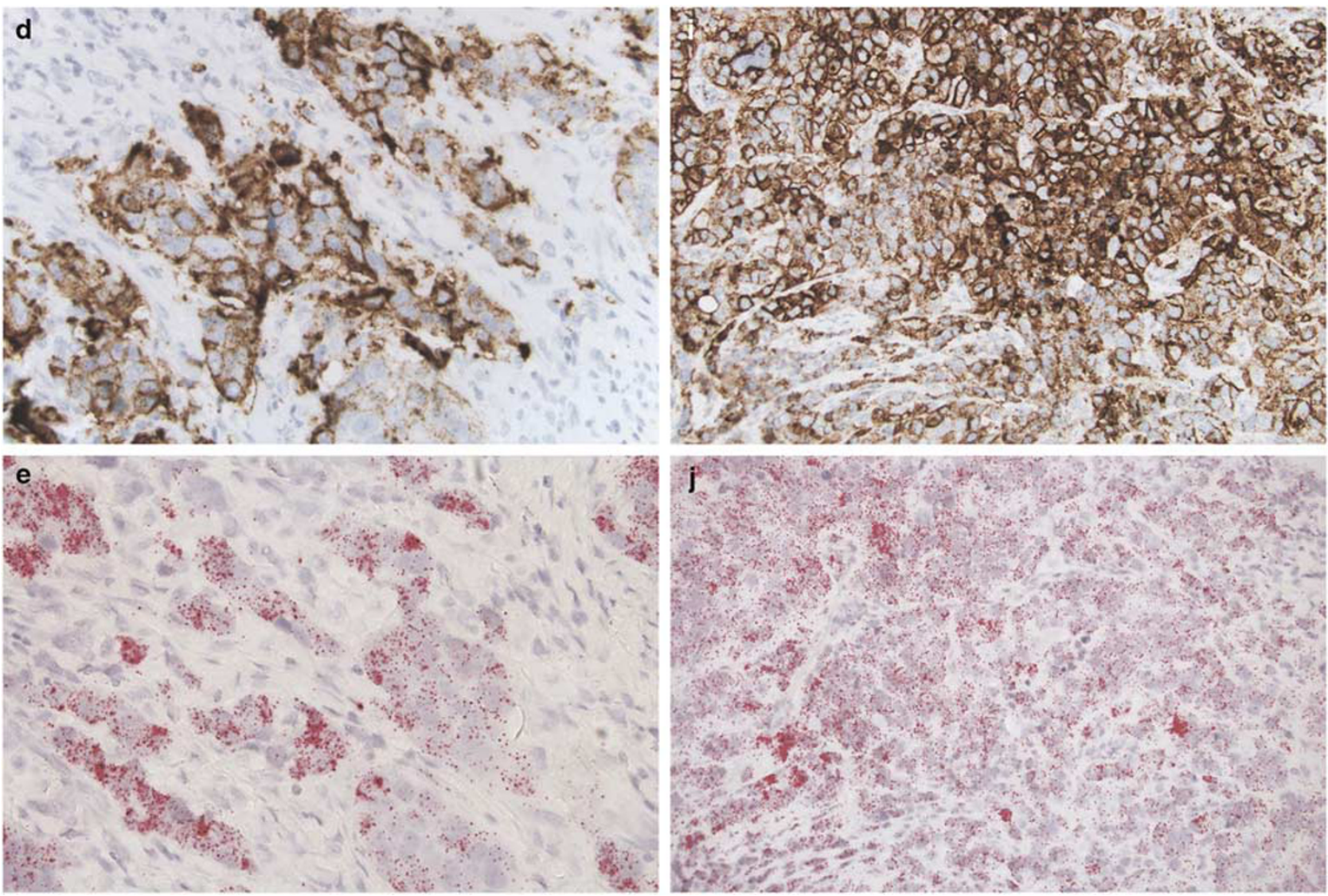

Figure 1 (Continued).

Table 1 Clinico-pathological characteristics of patient cohort

\begin{tabular}{|c|c|c|c|c|c|c|c|c|}
\hline $\begin{array}{l}\text { Clinico-pathological } \\
\text { feature }\end{array}$ & Level & $\begin{array}{l}\text { Overall no. } \\
\qquad \%)\end{array}$ & $\begin{array}{c}P D-L 1 \text { pos } \geq 1 \\
\text { marker }\end{array}$ & PD-L1 neg & $\mathrm{P}$ value & $\begin{array}{l}\text { SP142-IC } \\
\text { pos }\end{array}$ & SP142-IC neg & $\mathrm{P}$ value \\
\hline Age & Mean, s.d. & $67.9 \pm 11$ & $65.6 \pm 8.5$ & $68.5 \pm 11.6$ & 0.29 & $67 \pm 9.2$ & $68.1 \pm 11.7$ & 0.67 \\
\hline \multirow[t]{2}{*}{ Sex $(\%)$} & $\mathrm{F}$ & $35(35)$ & $8(38)$ & $27(34)$ & 0.91 & $7(26)$ & $27(38)$ & 0.40 \\
\hline & M & 66 (65) & $13(62)$ & $53(66)$ & & $20(74)$ & $45(63)$ & \\
\hline \multirow[t]{2}{*}{ Tumor type } & UC & $81(80)$ & $18(86)$ & 63 (79) & 0.83 & 24 (89) & $55(76)$ & 0.27 \\
\hline & Non-UC ${ }^{\mathrm{a}}$ & $20(20)$ & 3 (14) & $17(21)$ & & 3 (11) & $17(24)$ & \\
\hline Tumor size & Mean, s.d. & $4.27 \pm 2$ & $4.26 \pm 1.3$ & $4.27 \pm 2.2$ & 0.99 & $3.77 \pm 1.5$ & $4.54 \pm 2.2$ & 0.14 \\
\hline \multirow[t]{4}{*}{ Stage (1-4) } & $\leq \mathrm{pT} 1$ & $11(11)$ & $1(5)$ & $6(8)$ & 0.83 & $1(4)$ & $6(9)$ & 0.53 \\
\hline & pT2 & 17 (17) & $3(14)$ & 14 (18) & & $5(19)$ & 10 (15) & \\
\hline & pT3 & $47(47)$ & $12(57)$ & 35 (46) & & $15(58)$ & $32(46)$ & \\
\hline & pT4 & $26(26)$ & $5(24)$ & 21 (28) & & 5 (19) & 21 (30) & \\
\hline \multirow{2}{*}{ Stage low vs high } & $\leq \mathrm{pT} 2$ & $28(28)$ & $4(19)$ & 23 (29) & 0.52 & $7(26)$ & $18(25)$ & $>0.99$ \\
\hline & $\geq$ pT3 & $73(72)$ & $17(81)$ & $56(71)$ & & $20(74)$ & $53(75)$ & \\
\hline \multirow[t]{3}{*}{ LN status } & No & 15 (15) & 3 (14) & 12 (15) & 0.90 & $6(22)$ & 9 (13) & 0.49 \\
\hline & N1 & 27 (27) & $6(29)$ & $21(26)$ & & $8(30)$ & $19(26)$ & \\
\hline & N2 & 59 (58) & 12 (57) & 47 (59) & & $13(48)$ & $44(61)$ & \\
\hline \multirow[t]{2}{*}{ BCG treatment } & No & $72(73)$ & $16(76)$ & $56(72)$ & 0.90 & $18(67)$ & $52(74)$ & 0.62 \\
\hline & Yes & 27 (27) & $5(24)$ & $22(28)$ & & $9(33)$ & $18(26)$ & \\
\hline \multirow[t]{2}{*}{ Chemotherapy } & No & $21(30)$ & $3(20)$ & $18(33)$ & 0.53 & $5(24)$ & 16 (33) & 0.61 \\
\hline & Yes & $49(70)$ & $12(80)$ & 37 (67) & & $16(76)$ & 32 (67) & \\
\hline \multirow[t]{2}{*}{ Radiotherapy } & No & $62(92)$ & $13(87)$ & $49(94)$ & 0.67 & 17 (90) & $44(94)$ & 0.95 \\
\hline & Yes & $5(8)$ & $2(13)$ & $3(6)$ & & $2(11)$ & $3(6)$ & \\
\hline \multirow[t]{2}{*}{ Death } & No & 84 (84) & 19 (91) & 65 (82) & 0.56 & 25 (93) & 57 (80) & 0.24 \\
\hline & Yes & 16 (16) & $2(10)$ & 14 (18) & & $2(7)$ & $14(20)$ & \\
\hline
\end{tabular}

${ }^{\mathrm{a}}$ Non-UC cases included tumors with variant morphology such as micropapillary $(n=11)$, squamous differentiation $(n=2)$, glandular $(n=1)$, squamous and glandular $(n=1)$, plasmacytoid $(n=1)$, signet ring $(n=1)$, and adenocarcinoma $(n=3)$. 

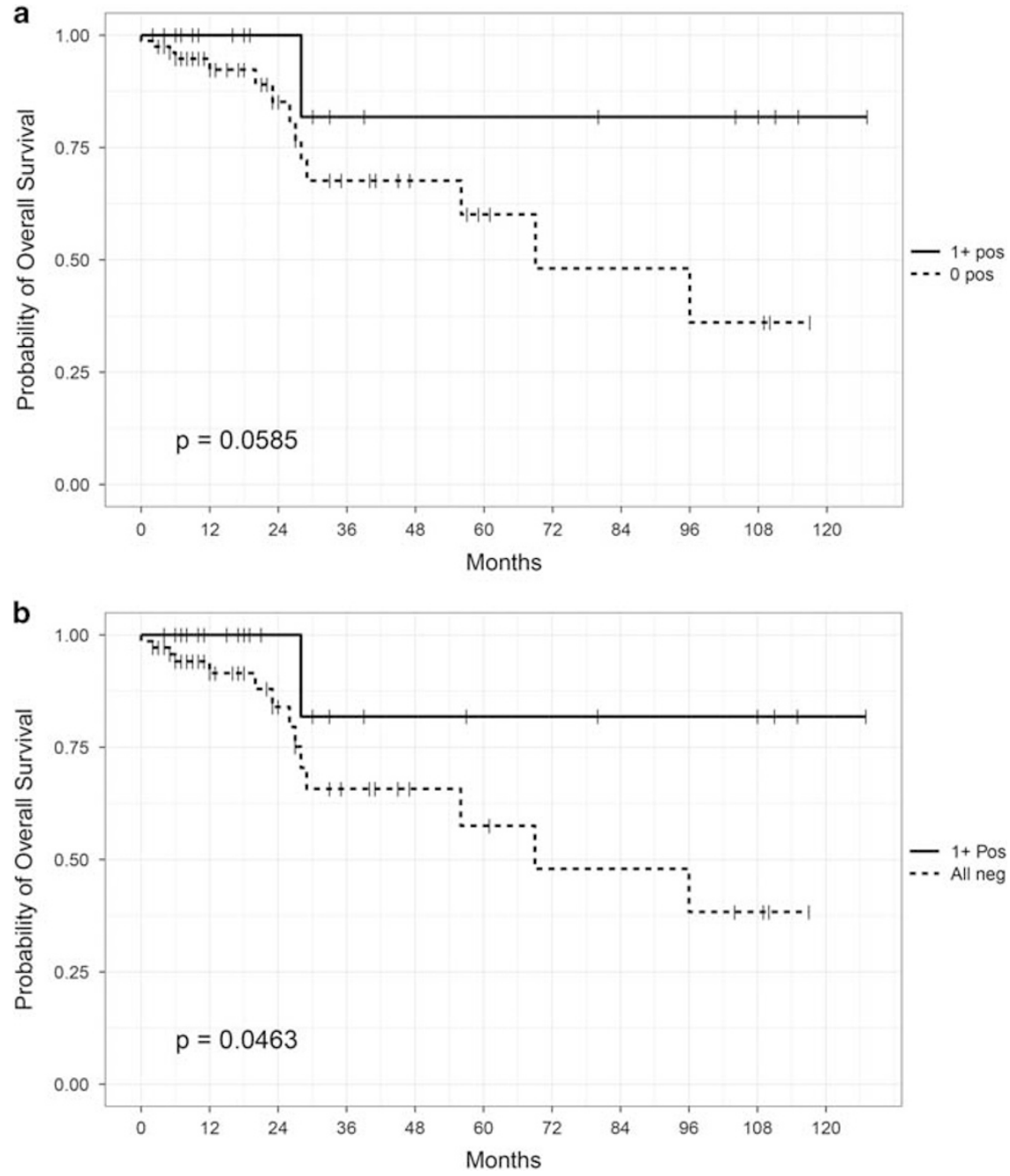

Figure 2 Kaplan-Meier plots for overall survival endpoint showing that patients with positive PD-L1 tumor cell expression by $\geq 1$ marker (solid line) have improved probability of OS vs patients with negative PD-L1 tumors (dashed line) in tumor cells (a) and in tumorinfiltrating immune cells by SP142 (b).

Table 2 Summary of concordance correlation coefficients of pairwise comparison of manual scores between four anti-PD-L1 antibodies in all 624 tissue array cores for tumor cells (A) and for tumor-infiltrating immune cells (B)

\begin{tabular}{|c|c|c|c|c|c|}
\hline Marker 1 & Marker 2 & Primary $U C(\mathrm{~N}=345)$ & LN metastases $(\mathrm{N}=231)$ & Distant metastases $(\mathrm{N}=48)$ & All data $(\mathrm{N}=624)$ \\
\hline Pairwise comparison & \multicolumn{5}{|c|}{ A. Tumor cells: CCC and its $95 \%$ confidence intervals, all cores $(\mathrm{N}=624)$} \\
\hline 22C3 & 28.8 & $0.84(0.80-0.86)$ & $0.97(0.96-0.97)$ & $0.99(0.98-0.99)$ & $0.88(0.86-0.90)$ \\
\hline 22C3 & E1L3N & $0.81(0.78-0.85)$ & $0.88(0.85-0.91)$ & $0.93(0.89-0.96)$ & $0.85(0.82-0.87)$ \\
\hline 22C3 & SP142 & $0.73(0.68-0.78)$ & $0.64(0.59-0.69)$ & $0.89(0.82-0.93)$ & $0.76(0.73-0.79)$ \\
\hline 28.8 & E1L3N & $0.90(0.88-0.92)$ & $0.83(0.80-0.86)$ & $0.94(0.90-0.96)$ & $0.90(0.89-0.91)$ \\
\hline 28.8 & SP142 & $0.87(0.85-0.89)$ & $0.55(0.49-0.61)$ & $0.89(0.82-0.93)$ & $0.85(0.83-0.87)$ \\
\hline E1L3N & SP142 & $0.85(0.83-0.88)$ & $0.73(0.69-0.77)$ & $0.97(0.95-0.98)$ & $0.87(0.85-0.89)$ \\
\hline Pairwise comparison & \multicolumn{5}{|c|}{ B. Immune cells: CCC and its 95\% confidence intervals, all cores $(\mathrm{N}=624)$} \\
\hline 22C3-IC & 28.8-IC & $0.84(0.80-0.87)$ & $0.89(0.85-0.91)$ & $0.95(0.92-0.97)$ & $0.85(0.83-0.97)$ \\
\hline 22C3-IC & E1L3N-IC & $0.69(0.64-0.74)$ & $0.85(0.81-0.88)$ & $0.72(0.58-0.83)$ & $0.74(0.71-0.78)$ \\
\hline 22C3-IC & SP142-IC & $0.34(0.25-0.42)$ & $0.49(0.39-0.57)$ & $0.15(0.08-0.22)$ & $0.35(0.29-0.40)$ \\
\hline 28.8-IC & E1L3N-IC & $0.77(0.73-0.81)$ & $0.84(0.80-0.87)$ & $0.84(0.74-0.91)$ & $0.79(0.77-0.82)$ \\
\hline 28.8-IC & SP142-IC & $0.35(0.27-0.43)$ & $0.60(0.52-0.67)$ & $0.17(0.09-0.24)$ & $0.38(0.32-0.43)$ \\
\hline E1L3N-IC & SP142-IC & $0.25(0.18-0.31)$ & $0.64(0.56-0.71)$ & $0.08(0.01-0.15)$ & $0.30(0.24-0.35)$ \\
\hline
\end{tabular}

IC, tumor-infiltrating immune cells 
Table 3 Summary of concordance correlation coefficients between protein expression combined scores and RNA expression levels by RNAscope in all 624 tissue array cores (A) and positive only cores (B)

\begin{tabular}{|c|c|c|c|c|c|}
\hline Marker 1 & Marker 2 & Primary $U C(\mathrm{~N}=345)$ & $L N$ metastases $(\mathrm{N}=231)$ & Distant metastases $(\mathrm{N}=48)$ & All data $(\mathrm{N}=624)$ \\
\hline Pairwise comparison & \multicolumn{5}{|c|}{ A. CCC and its $95 \%$ confidence intervals, all cores $(N=624)$} \\
\hline 22C3 & RNAscope & $0.74(0.69-0.78)$ & $0.65(0.57-0.71)$ & $0.90(0.84-0.93)$ & $0.75(0.72-0.78)$ \\
\hline 28.8 & RNAscope & $0.89(0.87-0.91)$ & $0.68(0.60-0.74)$ & $0.89(0.83-0.93)$ & $0.87(0.84-0.88)$ \\
\hline E1L3N & RNAscope & $0.77(0.73-0.81)$ & $0.61(0.53-0.67)$ & $0.94(0.90-0.97)$ & $0.77(0.74-0.80)$ \\
\hline SP142 & RNAscope & $0.74(0.69-0.78)$ & $0.37(0.30-0.44)$ & $0.93(0.87-0.96)$ & $0.72(0.69-0.76)$ \\
\hline Pairwise comparison & \multicolumn{5}{|c|}{ B. CCC and its $95 \%$ confidence intervals, positive cores by $\geq 1$ marker $(\mathrm{N}=132)$} \\
\hline Marker 1 & Marker 2 & Primary UC & LN metastases & Distant metastases & All data \\
\hline $22 \mathrm{C} 3$ & RNAscope & $0.66(0.55-0.75)$ & $0.42(0.14-0.64)$ & $0.55(0.11-0.87)$ & $0.66(0.56-0.74)$ \\
\hline 28.8 & RNAscope & $0.86(0.81-0.91)$ & $0.42(0.12-0.65)$ & $0.47(0.15-0.82)$ & $0.81(0.75-0.86)$ \\
\hline E1L3N & RNAscope & $0.71(0.62-0.79)$ & $0.42(0.17-0.62)$ & $0.87(0.37-0.98)$ & $0.70(0.61-0.77)$ \\
\hline SP142 & RNAscope & $0.68(0.57-0.76)$ & $0.21(0.04-0.37)$ & $0.79(0.16-0.96)$ & $0.64(0.55-0.72)$ \\
\hline
\end{tabular}

especially in metastatic sites (Supplementary Figure 3).

Pairwise comparison of PD-L1 expression on tumor-infiltrating immune cells showed high agreement between clones 22C3/28.8 (concordance correlation coefficient 0.85), 28.8/E1L3N (concordance correlation coefficient 0.79), and 28.8/E1L3N (concordance correlation coefficient 0.74) in all 624 cores. Concordance between SP142-IC and PD-L1 expression on immune cells by three other clones was substantially lower when assessed in all cores (range: 0.3-0.38), but showed higher concordance in regional lymph node metastases (range: 0.49-0.64) (Table 2b).

\section{Correlation Between Protein Expression Levels and RNA ISH in Tumor Cells}

The RNA ISH and protein expression levels in all 624 cores showed moderate to high correlation with concordance correlation coefficients ranging from 0.72 to 0.87 (Table 3). The level of agreement between protein and RNA ISH was highest for distant metastases (range: 0.89-0.94), intermediate for primary urothelial carcinomas (range: 0.74-0.89) and lowest for lymph node metastases (range: $0.37-$ 0.68). Comparison of RNAscope with four different antibodies showed highest concordance with clone 28.8 (range: 0.68-0.89) and lowest for clone SP142 (range: 0.37-0.93).

\section{Correlation Between PD-L1 Protein Expression Levels in Tumor Cells vs Immune Cells}

Frequency of PD-L1 expression in immune cells (\% positivity) assessed on SP142 immunostaining (SP142-IC) was $22.8 \%$ in primary urothelial carcinomas, $17.4 \%$ in lymph node mets and $27.1 \%$ in distant mets (Supplementary Table 2). Pairwise comparison of SP142-IC with tumor cell expression by the four markers showed only moderate concordance with concordance correlation coefficients ranging $0.55-0.74$ in all 624 cores, and $0.42-0.67$ in 132 positive cores (Supplementary Table 3). The highest pairwise agreement was between SP142-IC and SP142-tumor, whereas the lowest agreement was between SP142-IC and clones 22C3 and 28.8. Correlation was the lowest in lymph node metastases and distant metastases (range: 0.1-0.41).

\section{Intra-Tumor Heterogeneity of PD-L1 Protein and mRNA Expression}

The intra-tumor heterogeneity was assessed by comparing expression levels across tissue cores derived from the same tumor (Supplementary Table 4). The intraclass correlation coefficients for overall intra-tumor heterogeneity showed excellent concordance for all 4 PD-L1 clones in tumor cells (range: 0.90-0.94). PD-L1 intra-tumor heterogeneity was lowest in primary tumors (range: 0.95-0.98), and slightly higher in lymph nodes (range: 0.76-0.98) and distant metastases (range: 0.75-1). RNAscope results were highly concordant for both primary and metastatic sites (range: 0.87-0.98).

PD-L1 expression on tumor-infiltrating immune cells showed low heterogeneity for all four clones in primary tumors (range: 0.89-0.93) and lymph node metastases (range: 0.83-0.94), and slightly higher heterogeneity in distant metastases (range: 0.6-0.73).

\section{Manual Scoring vs Automated Image Analysis Agreement}

Comparison of manual scores and Aperio-generated combined scores showed excellent concordance for all tissue microarray cores (negative and positive) with concordance correlation coefficients ranging between 94 and 96\% agreement, and concordance correlation coefficients ranging between 92 and $96 \%$ among positive cores (Supplementary Table 5). However, concordance correlation coefficients in positive distant metastases was lower for clones 28.8 (concordance correlation coefficient: 0.62 ) and clone SP142 (concordance correlation coefficient: 0.79). This could be attributed to higher background staining for 28.8, and punctate staining pattern instead of continuous membranous pattern for clone 
SP142, which may have affected automated image analysis.

\section{Discussion}

PD-L1 immunohistochemical testing in cancer tissues suffer from lack of standardization and from limited validation, resulting in wide variety of assays that use different antibodies, reagents, staining platforms, and scoring methodologies. ${ }^{1,13}$ Rapidly developing immunotherapy requires large-scale quantitative validation studies comparing most commonly used PD-L1 immunoassays for concordance and accurate, reproducible measurement of targeted molecule. Our study is the first to address PD-L1 assay performance in large numbers of primary and metastatic bladder cancers, which will be increasingly tested and treated by check-point inhibitors following recent accelerated FDA approvals. $^{8}$

In the present high-throughput study, we investigated the four most commonly used anti-PD-L1 antibodies (3 FDA-approved). The prevalence of PD-L1 expression by $\geq 1$ marker in all bladder cancers $(N=235)$ was $23.8 \%$, with slightly higher prevalence in primary urothelial carcinomas vs regional lymph node or distant metastases. Positive staining with $\geq 2$ antibodies occurred in $21.1 \%$ primary, $15.6 \%$ regional metastases, and $12.5 \%$ distant metastases, nearly as often as with at least one antibody. The proportion of strongly positive cases was lower in primary tumors $(34.1 \%)$ than in metastatic sites (46.7\%). Importantly, we found high concordance between PD-L1 expression in matched primary and metastatic urothelial carcinomas with agreement in $89.9 \%$ cases, although the majority of our matched cases were obtained from patients with regional metastases excised at the time of primary tumor resection. Our findings in primary urothelial carcinomas are consistent with previously reported prevalence rates of PD-L1 expression in tumor cells ranging from 14 to $28 \% .^{4,20-23}$ To our knowledge we are the first to report prevalence of PD-L1 expression in regional and distant urothelial carcinoma metastases.

We found high concordance between the four immunoassays in tumor cells despite the differences in antibody design, molecular properties, antigen retrievals, detection systems, and staining platforms. Similar finding of minimal inter-assay variability were reported in lung cancer for PD-L expression using various combinations of clones 22C3, 28.8, SP142, E1L3N, 9A11, and SP263. ${ }^{14,15,24}$ E1L3N rabbit monoclonal antibody is the only one out of four studied here antibodies that is not FDAapproved, platform-dependent or linked to a specific pharmacological agent, although it is one of the most frequently used research antibodies. ${ }^{4}$ Our comprehensive concordance study supported by several other reports provide strong evidence that E1L3N clone is as accurate and reproducible in detecting PD-L1 protein in urothelial carcinomas as any other FDA-approved immunoassay. ${ }^{5,15,24-26}$ To avoid subjectivity in scoring, we scored all immunostains following same approach resulting in highly concordant results between manual scores and automated scores for all four immunomarkers (concordance correlation coefficients: 0.92-0.97). A recent study comparing four scoring methods showed that percentage of tumor cell staining at any intensity has similar prognostic value as more complex scoring methods, and incorporation of immune cell staining did not improve predictive value of PD-L1 (clone 22C3) in lung cancer. ${ }^{27}$

We found that patients with PD-L1 positive tumors by $\geq 1$ marker and with PD-L1 negative tumors were similar in age, sex, histopathological, and clinical parameters. However, presence of any PD-L1 expression in tumor cells was associated with improved overall survival $(P=0.058)$. PD-L1 expression in tumor-infiltrating immune cells was present in 27 tumors and also was associated with improved outcomes $(P=0.046)$. The overall impact of PD-L1 expression on prognosis remains controversial likely due to variability of methodologies for evaluating PD-L1 expression and its scoring. ${ }^{6,20-23}$ Taube et $a 2^{28}$ found that PD-L1 staining of tumor cells in various tumors was more predictive of response to anti-PD-1 check-point blockade than PD-L1 expression on immune cells. However, for bladder cancer it remains unclear whether PD-L1 expression in tumor cells or in immune cells is more predictive of outcome and treatment response. .,3,7,11,21,26 $^{-1}$

On May 2016, FDA granted accelerated approval for atezolizumab (Tecentriq) to treat patients with locally advanced or metastatic urothelial carcinomas after platinum-containing chemotherapy. ${ }^{8}$ The objective response rates were increased in patients with $>5 \%$ immune cells by SP142 assay; however, this agreement was modest and some complete responders were reported with negative SP142-IC staining. Similar to atezolizumab antitumor activity has been reported for other PD-1 or PD-L1-targeted products (eg, avelumab, durvalumab, pembrolizumab, and nivolumab) in advanced urothelial carcinoma. ${ }^{8,9,26,29}$ Not surprisingly, on May 2017, FDA granted two accelerated approvals for pembrolizumab (Keytruda) and durvalumab (Imfinzi) to treat unresectable and metastatic solid tumors that have progressed following prior treatment (https:// www.fda.gov). The objective response rates in populations of 30-300 study patients were 15-52\% (mean 30\%) including some durable responses with good tolerance and suggesting a class effect of PD-1/ PD-L1-targeted products in the disease. ${ }^{3,10,26}$

It is important to emphasize that our study showed lower sensitivity and higher intra-tumor heterogeneity of SP142 immunoassay compared to other clones in both tumor cells and immune cells, as well as only modest agreement between PD-L1 expression SP142IC vs tumor cell expression by all four markers 
(concordance correlation coefficients: 0.55-0.73). The same observation was made in 'Blueprint PDL1 immunohistochemical assay comparison project'. ${ }^{14}$ From a pathologist perspective, separate interpretation of tumor cell and immune cell expression for clone SP142 is cumbersome and poses significant challenge in some cases. Moreover, unusual punctate, dot-like, or clump-like expression pattern instead of membranous pattern seen with other clones could prevent from reproducible interpretation of SP142 staining results. ${ }^{30}$ Further studies addressing intriguing differences in assay sensitivity, expression heterogeneity and post-analytical variables unique to clone SP142 are warranted. Our findings also suggest that PD-L1 immunoassays other than SP142 could be utilized and should be tested for predicting durable objective response rates in patients with advanced bladder cancer.

Our study has several limitations including its retrospective nature and utilization of tissue microarrays without whole-section analysis. Recent study of conventional tissue sections revealed substantial heterogeneity of PD-L1 expression in lung cancer and resulted in higher variation of immunohistochemical results. ${ }^{31}$ On the other hand, in clinical practice, PD-L1 assessment is frequently done on small archival tissue samples introducing preanalytical variables and sampling error similar to tissue microarray cores. Although we found that PD-L1 positivity is associated with overall survival and may serve as a prognostic marker, no conclusion could be made regarding specific treatment response as the majority of our patients had locally advanced disease with only few distant metastases, and none were treated with PD-1 axis inhibitors.

PD-L1 mRNA expression was used as a molecular correlate for PD-L1 immunoassays; however, it remains unclear whether mRNA expression could be viewed as a gold standard for PD-L1 overexpression. ${ }^{13,15}$ We postulated that RNA expression values, as measured by RNA ISH (RNAscope), could potentially serve as a relevant inter-method comparator. To our knowledge, RNA ISH has not been clinically or analytically validated in this context, and a variety of factors, including posttranslational protein modification, could yield discordant results between RNA and protein expression levels. Therefore, it would be premature to suggest that RNA ISH represents a gold standard test for PDL1 assays. However, we believe the comparison of RNA and protein expression is of potential value in normalizing the results of immunohistochemical assays, generally, although further study is required to establish the performance characteristics of RNA ISH for PD-L1. In light of these considerations, it is of interest that our results indicate rather robust concordance between the two methods.

Similar performance of four immunohistochemical assays as documented by our study could potentially eliminate several issues-related PD-L1 laboratory testing when neither an ordering clinician nor pathologist can make an informed decision about test of choice. It could also potentially reduce the cost of PD-L1 testing for the patient; prevent laboratories from running multiple antibody panels and from running expensive tests where payers may not be compensated. However, slightly higher prevalence of PD-L1 expression by any one antibody vs $\geq 2$ antibodies may justify a utilization of antibody panels in cases with borderline low-expression levels at the detection limit of immunoassay.

In summary, PD-L1 is expressed in a substantial proportion of primary and metastatic urothelial carcinomas in both tumor cell membranes and tumor-infiltrating immune cells. Standardized manual scoring allowed comparison of four most commonly used antibodies and RNA ISH assay showing concordant results between different antibody clones, protein and RNA expression levels, and near equivalence of manual and automated scoring.

\section{Disclosure/conflict of interest}

The authors declare no conflict of interest.

\section{References}

1 Patel SP, Kurzrock R. PD-L1 expression as a predictive biomarker in cancer immunotherapy. Mol Cancer Ther 2015;14:847-856.

2 Topalian SL, Taube JM, Anders RA, et al. Mechanismdriven biomarkers to guide immune checkpoint blockade in cancer therapy. Nat Rev Cancer 2016;16: 275-287.

3 Bellmunt J, Powles T, Vogelzang NJ. A review on the evolution of PD-1/PD-L1 immunotherapy for bladder cancer: the future is now. Cancer Treat Rev 2017;54: 58-67.

4 Inaguma S, Wang Z, Lasota J, et al. Comprehensive immunohistochemical study of mesothelin (MSLN) using different monoclonal antibodies 5B2 and MN-1 in 1562 tumors with evaluation of its prognostic value in malignant pleural mesothelioma. Oncotarget 2017;8: 26744-26754.

5 Mahoney KM, Sun H, Liao X, et al. PD-L1 antibodies to its cytoplasmic domain most clearly delineate cell membranes in immunohistochemical staining of tumor cells. Cancer Immunol Res 2015;3:1308-1315.

6 Wang X, Teng F, Kong L, et al. PD-L1 expression in human cancers and its association with clinical outcomes. OncoTargets Ther 2016;9:5023-5039.

7 Balar AV, Galsky MD, Rosenberg JE, et al. Atezolizumab as first-line treatment in cisplatin-ineligible patients with locally advanced and metastatic urothelial carcinoma: a single-arm, multicentre, phase 2 trial. Lancet 2017;389:67-76.

8 Ning YM, Suzman D, Maher VE, et al. FDA approval summary: atezolizumab for the treatment of patients with progressive advanced urothelial carcinoma after platinum-containing chemotherapy. Oncologist 2017;22: 743-749.

9 Powles T, Eder JP, Fine GD, et al. MPDL3280A (antiPD-L1) treatment leads to clinical activity in metastatic bladder cancer. Nature 2014;515:558-562. 
10 Rosenberg JE, Hoffman-Censits J, Powles T, et al. Atezolizumab in patients with locally advanced and metastatic urothelial carcinoma who have progressed following treatment with platinum-based chemotherapy: a single-arm, multicentre, phase 2 trial. Lancet 2016;387:1909-1920.

11 Gandini S, Massi D, Mandala M. PD-L1 expression in cancer patients receiving anti PD-1/PD-L1 antibodies: a systematic review and meta-analysis. Crit Rev Oncol Hematol 2016;100:88-98.

12 Ettinger DS, Wood DE, Akerley W, et al. NCCN guidelines insights: non-small cell lung cancer version 4.2016. J Natl Compr Cancer Netw 2016;14:255-264.

13 Kerr KM, Hirsch FR. Programmed death ligand-1 immunohistochemistry: friend or foe? Arch Pathol Lab Med 2016;140:326-331.

14 Hirsch FR, McElhinny A, Stanforth D, et al. PD-L1 immunohistochemistry assays for lung cancer: results from phase 1 of the blueprint PD-L1 IHC assay comparison project. J Thorac Oncol 2017;12:208-222.

15 Sheffield BS, Fulton R, Kalloger SE, et al. Investigation of PD-L1 biomarker testing methods for PD-1 axis inhibition in non-squamous non-small cell lung cancer. J Histochem Cytochem 2016;64:587-600.

16 Lin L, Hedayat S, Sinha B, et al. Statistical methods in assessing agreement: models, issues, and tools. Journal of the Am erican Stat istical Association 2002;97:257-270.

17 R Core Team. R: A Language and Environment for Statistical Computing. R Foundation for Statistical Computing: Vienna, Austria, 2016.

18 Stevenson M. epiR: Tools for the Analysis of Epidemiological Data. R package version 0.9-79, 2016.

19 Wolak ME, Fairbairn DJ, Paulsen YR. Guidelines for estimating repeatability. Methods Ecol Evol 2012;3: 129-137.

20 Inman BA, Sebo TJ, Frigola X, et al. PD-L1 (B7-H1) expression by urothelial carcinoma of the bladder and BCG-induced granulomata: associations with localized stage progression. Cancer 2007;109:1499-1505.

21 Bellmunt J, Mullane SA, Werner L, et al. Association of PD-L1 expression on tumor-infiltrating mononuclear cells and overall survival in patients with urothelial carcinoma. Ann Oncol 2015;26:812-817.
22 Faraj SF, Munari E, Guner G, et al. Assessment of tumoral PD-L1 expression and intratumoral CD8+ $\mathrm{T}$ cells in urothelial carcinoma. Urology 2015;85: 703.e1-703.e6.

23 Baras AS, Drake C, Liu JJ, et al. The ratio of CD8 to Treg tumor-infiltrating lymphocytes is associated with response to cisplatin-based neoadjuvant chemotherapy in patients with muscle invasive urothelial carcinoma of the bladder. Oncoimmunology 2016;5:e1134412.

24 Gaule P, Smithy JW, Toki M, et al. A quantitative comparison of antibodies to programmed cell death 1 ligand 1. JAMA Oncol 2017;3:256-259.

25 Cogswell J, Inzunza $\mathrm{HD}, \mathrm{Wu} \mathrm{Q}$, et al. An analytical comparison of Dako 28-8 PharmDx assay and an E1L3N laboratory-developed test in the immunohistochemical detection of programmed death-ligand 1. Mol Diagn Ther 2017;21:85-93.

26 Le Goux C, Damotte D, Vacher S, et al. Correlation between messenger RNA expression and protein expression of immune checkpoint-associated molecules in bladder urothelial carcinoma: a retrospective study. Urol Oncol 2017;35:257-263.

27 Dolled-Filhart M, Roach C, Toland G, et al. Development of a companion diagnostic for pembrolizumab in nonsmall cell lung cancer using immunohistochemistry for programmed death ligand-1. Arch Pathol Lab Med 2016;140:1243-1249.

28 Taube JM, Klein A, Brahmer JR, et al. Association of PD-1, PD-1 ligands, and other features of the tumor immune microenvironment with response to anti-PD-1 therapy. Clin Cancer Res 2014;20:5064-5074.

29 Plimack ER, Dunbrack RL, Brennan TA, et al. Defects in DNA repair genes predict response to neoadjuvant cisplatin-based chemotherapy in muscle-invasive bladder cancer. Eur Urol 2015;68:959-967.

30 Rehman JA, Han G, Carvajal-Hausdorf DE, et al. Quantitative and pathologist-read comparison of the heterogeneity of programmed death-ligand 1 (PD-L1) expression in non-small cell lung cancer. Mod Pathol 2017;30:340-349.

31 McLaughlin J, Han G, Schalper KA, et al. Quantitative assessment of the heterogeneity of PD-L1 expression in non-small-cell lung cancer. JAMA Oncol 2016;2:46-54.

Supplementary Information accompanies the paper on Modern Pathology website (http://www.nature.com/ modpathol) 